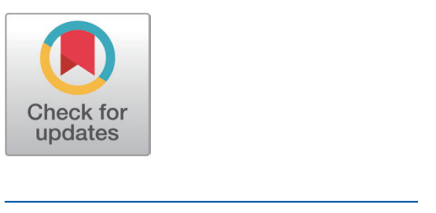

Received: Dec 2, 2021

Revised: Jan 5, 2022

Accepted: Jan 10, 2022

${ }^{*}$ Corresponding author Qiuye Lin

College of Food Science and

Technology, Yunnan Agricultural

University, Kunming 650201, China.

Tel: + 86-871-6522-7788

E-mail: linqiuye@126.com

Copyright $@ 2022$ Korean Society of

Animal Sciences and Technology.

This is an Open Access article

distributed under the terms of the

Creative Commons Attribution

Non-Commercial License (http://

creativecommons.org/licenses/by-

$\mathrm{nc} / 4.0 /$ ) which permits unrestricted

non-commercial use, distribution, and

reproduction in any medium, provided

the original work is properly cited.

ORCID

Shujuan Yang

https://orcid.org/0000-0002-8494-1128

Chenglin Deng

https://orcid.org/0000-0001-6386-228X

Yao Li

https://orcid.org/0000-0003-0699-1792

Weicheng $\mathrm{Li}$

https://orcid.org/0000-0001-9880-9390

Qiong Wu

https://orcid.org/0000-0002-4657-9877

Zhihong Sun

https://orcid.org/0000-0002-7605-2048

Zhenhui Cao

https://orcid.org/0000-0002-0063-4316

Qiuye Lin

https://orcid.org/0000-0002-8287-0047

\section{Complete genome sequence of Lactiplantibacillus plantarum ST, a potential probiotic strain with antibacterial properties}

\author{
Shujuan Yang ${ }^{1}$, Chenglin Deng ${ }^{2}$, Yao $\mathrm{Li}^{2}$, Weicheng $\mathrm{Li}^{1}$, Qiong $\mathrm{Wu}^{1}$, \\ Zhihong Sun ${ }^{1}$, Zhenhui Cao ${ }^{3}$ and Qiuye Lin ${ }^{2 *}$
}

${ }^{1}$ Key Laboratory of Dairy Biotechnology and Engineering, Ministry of Education of China, Inner Mongolia Agricultural University, Hohhot 010018, China

${ }^{2}$ College of Food Science and Technology, Yunnan Agricultural University, Kunming 650201, China

${ }^{3}$ Faculty of Animal Science and Technology, Yunnan Agricultural University, Kunming 650201, China

\begin{abstract}
Lactiplantibacillus plantarum (L. plantarum) ST was isolated from De'ang pickled tea in Yunnan Province, China. The genomes of strain ST were fully sequenced and analyzed using the PacBio RS II sequencing system. Our previous study has shown that $L$. plantarum ST is a potential probiotic strain. It had strong tolerance in the simulated artificial gastrointestinal tract, and in the antagonism tests, this strain showed strong antibacterial activity. Therefore, as a probiotic, it may be used in animal breeding. L. plantarum ST genome was composed of 1 circular chromosome and 7 plasmids. The length of the whole genome was $3320817 \mathrm{bp}$, and the annular chromosome size was $3058984 \mathrm{bp}$, guanine + cytosine ( $\mathrm{G} \pm \mathrm{C}$ ) content (\%) was $44.76 \%$, which contained 2945 protein-coding sequences (CDS). This study will contribute to a further comprehensive understanding of $L$. Plantarum ST at the genomic level and provide a theoretical basis for its future application in animal breeding.

Keywords: Lactiplantibacillus plantarum, Pickled tea, Whole-genome sequencing, Probiotics, Feed
\end{abstract}

Lactiplantibacillus plantarum (L. plantarum) used belongs to the genus Lactobacillus, which exists widely in a variety of environments and has been consumed in the context of fermented foods or used by humans for thousands of years. It is a recognized safe microorganism [1]. L. plantarum has many functions, such as maintaining the balance of intestinal flora, improving immunity and promoting nutrient absorption [2]. Studies have shown that dietary L. plantarum can improve the daily gain weight and daily feed intake of weaned piglets, as well as the feed conversion rate [3]. $L$. plantarum can also regulate the intestinal flora of broilers and accelerate the maturation of broilers [4]. According to previous studies [5], L. plantarum ST isolated from De'ang pickled tea, a traditional food consumed by the De' ang nationality of Yunnan, China. The probiotic characteristics of this strain were evaluated from several aspects. It was found that $L$. plantarum ST had good tolerance to gastric and intestinal fluid, strong adhesion and automatic aggregation ability in the intestinal tract, and exhibited antagonistic activity against Salmonella typhimurium and Escherichia coli. Therefore, it is considered that 
Competing interests

No potential conflict of interest relevant to this article was reported.

\section{Funding sources}

This study was supported by grants from the National Natural Science Foundation of China (NSFC) (31760448), Young Top-notch Talent of Yunnan Support Project for of High Level Talents (YNWR-QNBJ-2018-137), Yunnan Workstation of Sun Zhihong Expert (202105AF150055) and Major project of Natural Science Foundation of Inner Mongolia (2020ZD12).

\section{Acknowledgements}

Not applicable.

Availability of data and materia Upon reasonable request, the datasets of this study can be available from the corresponding author.

Authors' contributions

Conceptualization: Yang S, Lin Q.

Data curation: Yang S.

Formal analysis: Yang S, Deng C, Li Y, Wu Q, Cao Z.

Methodology: Yang S, Deng C, Li W, Wu Q, Sun Z, Lin Q.

Software: Yang S, Deng C, Li Y, Li W, Sun Z. Validation: Yang S, Deng C, Lin Q. Investigation: Yang S, Li Y, Lin Q.

Writing - original draft: Yang S.

Writing - review \& editing: Yang S, Deng C,

Li Y, Li W, Wu Q, Sun Z, Cao Z, Lin Q.

Ethics approval and consent to participate This article does not require IRB/IACUC approval because there are no human and animal participants. this strain has good probiotic characteristics. At the same time, our previous study has shown that dietary replacement of fermented soy bean hull and canola seed cake with $A$. niger and L. plantarum ST increased body weight and daily weight gain of 56-day-age Chahua broiler, a local chicken breed in Yunnan of China, compared to that prepared with Aspergillus niger alone (unpublished data). Therefore, $L$. plantarum ST shows good potential probiotic properties and it maybe can used as a probiotics in animal breeding.

Based on previous studies, the whole genome of L. plantarum ST was analyzed in this paper. L. plantarum ST was cultured in MRS medium (Guangzhou Huankai Microbial Technology, Guangzhou, China) at $37^{\circ} \mathrm{C}$ for 24 hours. Genomic DNA was extracted from the collected samples using the Wizard ${ }^{\circledR}$ Genomic DNA Purification Kit. PacBio SMRT sequencing process was used to establish a 10-kb library of extracted genomic DNA, after which the whole genome was sequenced using PacBio RS II (Pacific Biosciences, Menlo Park, CA, USA) sequencing platform [6]. L. plantarum ST sequence quality control and strain genome assembly were performed by RS_HGAP_Assembly.3 software in SMRT ${ }^{\circledR}$ Portal (V2.7). Circlator (V1.5.5) [7] was used to cyclize the assembled genome. Upload the assembled L. plantarum ST nucleic acid sequence file to EggNOG database (http://eggnog5.embl.de/) for genome function annotation. Upload the whole genome sequence of L. plantarum ST to CGview server (http://stothard.afns.ualberta.ca/cgview_ server/) to complete the genome circle map.

Data evaluation and sequence assembly were conducted for the sequenced sample L. plantarum ST to obtain the genome assembly results (Table 1), and the assembled sequences were spliced into a genome circle map (Fig. 1). The results showed that the L. plantarum ST genome had 1 circular chromosome and 7 plasmids. The whole genome length was $3320817 \mathrm{bp}$, in which the size of circular chromosome was 3058984 bp and guanine + cytosine $(\mathrm{G} \pm \mathrm{C})$ content (\%) was 44.76\%, which contained 2945 protein-coding sequences (CDS). Clusters of Orthologous Groups (COG) annotation results showed that these genes were clustered in 22 functional categories of protein (Fig. 2), among which the number of genes involved in carbohydrate transport and metabolism was the largest, suggesting that this strain has strong carbohydrate metabolism ability. The comparative genomics study of 114 strains of $L$. plantarum conducted by Mao et al. [8] demonstrated that the core functions of these strains were concentrated on carbohydrate metabolism and amino acid metabolism, which provide molecular support for strains to metabolize multiple sugars. Moreover, the ability of $L$. plantarum strains to utilize carbohydrates has been correlated with the carbohydrate metabolism-related genes. The COG annotation findings of 126 strains of $L$. plantarum in another study [9] revealed that the number of coding genes involved in carbohydrate metabolism was the largest, which was consistent with the COG annotation results in this study. Because

Table 1. Statistics of assembly results of Lactiplantibacillus plantarum ST genome

\begin{tabular}{clccc}
\hline Strains & Features & Number of scaffolds & Total Length / bp & G \pm C content $\%$ \\
\hline Plantarum ST & Chromosome & 1 & $3,058,984$ & 44.76 \\
& Plasmid1 & 1 & 64,531 & 39.75 \\
& Plasmid2 & 1 & 18,420 & 40.80 \\
& Plasmid3 & 1 & 14,663 & 34.04 \\
& Plasmid4 & 1 & 59,781 & 42.19 \\
& Plasmid5 & 1 & 38,169 & 40.38 \\
& Plasmid6 & 1 & 34,578 & 38.02 \\
& Plasmid7 & 1 & 31,691 & 37.51 \\
\hline
\end{tabular}

G, guanine; C, cytosine. 

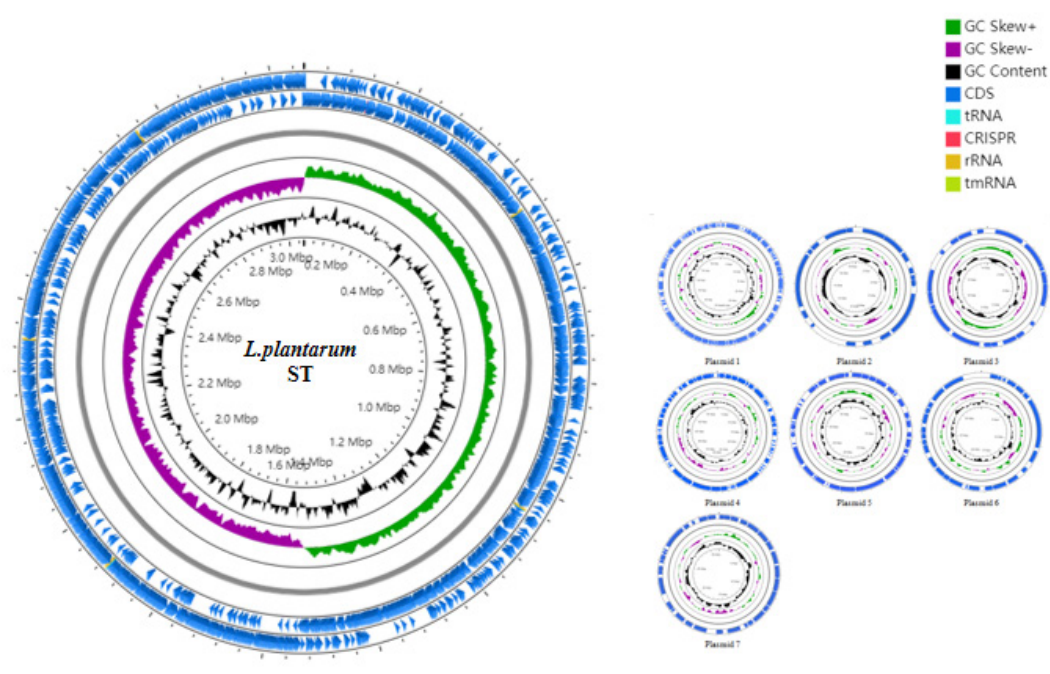

Fig. 1. Circular map of Lactiplantibacillus plantarum ST genome. G, guanine; C, cytosine; CDS, coding sequences.

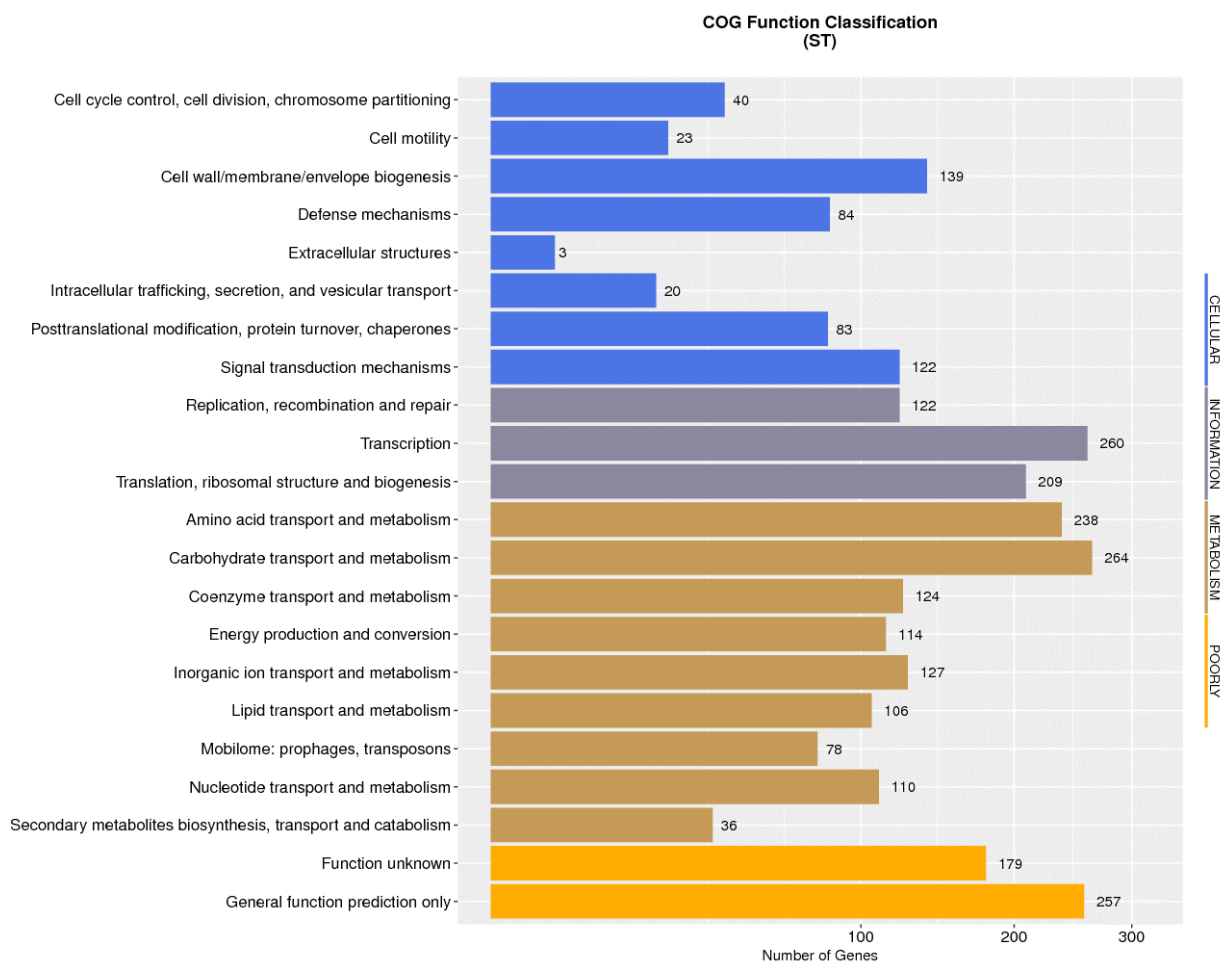

Fig. 2. COG Functional annotation of Lactiplantibacillus plantarum ST. COG, clusters of orthologous groups.

carbohydrates are the main energy source to support growth and development of microorganisms, lactic acid bacteria can adapt to the environment specifically through obtaining carbohydrates from environment and/or removing unnecessary carbohydrate genes.

In addition, the genome of ST strain contained 139 genes annotated with cell wall, cell membrane and extracellular membrane biosynthesis, which can provide evidence that this strain has 
strong biofilm formation ability, and in turn facilitate resistance against external unfavorable factors. Eighty-four annotated genes were identified in the defense mechanism, indicating that the strain could resist the digestive tract environment and provide necessary conditions for stable colonization in intestinal epithelial cells. The defense mechanism also contained four functional sequences synthesizing $\mathrm{ABC}$ antibacterial peptide transport system, which is recognized as a necessary factor for antibacterial peptide transport and secretion, and ensure the antibacterial characteristics of the strain. The genomic information of $L$. plantarum ST provides scientific basis for its future application in animal breeding.

\section{NUCLEOTIDE SEQUENCE ACCESSION NUMBER}

L. plantarum ST complete genome sequence has been submitted to GenBank database (https:// www.ncbi.nlm.nih.gov/), and deposited under the BioProjecct PRJNA792432.

\section{REFERENCES}

1. Cebeci A, Gürakan C. Properties of potential probiotic Lactobacillus plantarum strains. Food Microbiol. 2003;20:511-8. https://doi.org/10.1016/S0740-0020(02)00174-0

2. Siezen RJ, van Hylckama Vlieg JET. Genomic diversity and versatility of Lactobacillus plantarum, a natural metabolic engineer. Microb Cell Fact. 2011;10 Suppl 1:S3. https://doi. org/10.1186/1475-2859-10-S1-S3

3. Yang Y, Pan B, Sun X. The function of Lactobacillus plantarum and its application in animal production. Feed Res. 2013;2:36-7.https://doi.org/10.3969/j.issn.1002-2813.2013.02.013

4. Gao P, Ma C, Sun Z, Wang L, Huang S, Su X, et al. Feed-additive probiotics accelerate yet antibiotics delay intestinal microbiota maturation in broiler chicken. Microbiome. 2017;5:91. https://doi.org/10.1186/s40168-017-0315-1

5. Cao Z, Pan H, Li S, Shi C, Wang S, Wang F, et al. In vitro evaluation of probiotic potential of lactic acid bacteria isolated from Yunnan De'ang pickled tea. Probiotics Antimicrob Proteins. 2019;11:103-12. https://doi.org/10.1007/s12602-018-9395-x

6. Zhong Z, Sun TS, Chen YF. Genomic analysis revealed the molecular mechanism of extracellular polysaccharide enrichment in Streptococcus thermophilus ND-07. China Dairy Ind. 2018;46:9-11.

7. Goris J, Konstantinidis KT, Klappenbach JA, Coenye T, Vandamme P, Tiedje JM. DNA-DNA hybridization values and their relationship to whole-genome sequence similarities. Int J Syst Evol Microbiol. 2007;57:81-91. https://doi.org/10.1099/ijs.0.64483-0

8. Mao B, Yin R, Li X, Cui S, Zhang H, Zhao J, et al. Comparative genomic analysis of Lactiplantibacillus plantarum isolated from different niches. Genes. 2021;12:241. https://doi. org/10.3390/GENES12020241

9. Liu YH, Li WC, Yu ZJ, Zhao FY, Zhao J, Sun Z, et al. Population genome of Lactobacillus plantarum isolated from different environments. Microbiol China. 2019;46:3388-401. https:// doi.org/10.13344/j.microbiol.china.190132 